\title{
Coeffects of diet and neonicotinoid exposure on honeybee mobility and food choice
}

\author{
Chiara Vodovnik ${ }^{1}$, Anna-Maria Borshagovski ${ }^{2}$, Sanja Maria Hakala ${ }^{3}$, \\ Matti LEPONIEMI ${ }^{1}$, Dalial FreITAK ${ }^{1}$ iD \\ ${ }^{1}$ Institute of Biology, University of Graz, Graz, Austria \\ ${ }^{2}$ Department of Ecology and Genetics, University of Oulu, Oulu, Finland \\ ${ }^{3}$ Faculty of Biological and Environmental Sciences, University of Helsinki, Helsinki, Finland
}

Received 25 August 2020 - Revised 4 February 2021 - Accepted 24 February 2021

\begin{abstract}
Malnutrition and pesticide exposure are severe factors contributing to the current losses of honeybee colonies. As these stressors often occur combined, we studied the synergistic effects of different diets and pesticide exposure on food choice and mobility of Apis mellifera. We fed beehives with different food sources and exposed the bees to sublethal doses of thiacloprid. After that three different types of honey and pollen were offered in separate choice assays and behaviour towards food was recorded. Thiacloprid significantly affected the food choice in the honey assay, increasing the bees' preference of polyfloral honey, while pre-pesticide diet had no influence. The pollen choice remained similar regardless of treatments, as bees always preferred polyfloral pollen. Interestingly, pesticide exposure affected bee mobility differently, depending on the previous diet. These results indicate that the diet is an important factor influencing the susceptibility to pesticides.
\end{abstract}

\section{Thiacloprid / Apoidea / Food preference / Malnutrition / Behaviour}

\section{INTRODUCTION}

Honeybees contribute to ecosystem services by pollinating a large variety of wild and cultivated plants on which other biological processes and service-providing organisms depend on (Kremen et al. 2007). In recent years, however, losses of managed colonies have been recorded all over the world (Steinhauer et al. 2018; Requier et al. 2018; van Engelsdorp and Meixner 2010). The reasons for this are manifold and include diseases, constant intensification of agriculture, increase of monocultures, and the closely linked excessive use of agrochemicals, all together leading to habitat loss and a reduced availability of a balanced nutrition (Goulson et al. 2015).

Corresponding author: D. Freitak, dalial.freitak@unigraz.at

Manuscript editor: Cedric Alaux
Neonicotinoids are currently the most used insecticides in the world (Craddock et al. 2019). They are systemic pesticides, implying that they end up in all parts of the plants, including pollen, nectar, and guttation fluids. This allows an effective and holistic protection against herbivores, but also means that non-target organisms like pollinators are exposed to the neurotoxic compounds. Neonicotinoids are water-soluble and highly persistent chemicals, so that residues can still be found in the environment even after longer periods of non-application. Neonicotinoids bind to nicotinic acetylcholine receptors (nAChRs), which affect the central nervous system of insects (Goulson 2013). The concentrations present in pollen and nectar may not cause direct mortality, but even sublethal doses can have far-reaching effects on the orientation and memory of honeybees (Yang et al. 2008), therefore endangering the 
individuals' foraging-ability and affecting the survival of the whole colony (Henry et al. 2012).

A balanced nutrition is a critical factor influencing honeybee health: Nectar provides bees with important vitamins, minerals, and lipids and proteins in pollen comprise amino acids vital for the development of individuals (Brodschneider and Crailsheim 2010). Poor nutrition can have a bearing on the lifespan, as well as on the resilience against potential pathogens (Dolezal and Toth 2018). The lack of diverse food resources, to which bees are increasingly exposed in the modern monocultural farming lands, therefore may play a key role in the ongoing weakening of colonies (Di Pasquale et al. 2013).

Since little is known about the synergistic or additional effects of an inadequate diet and pesticide exposure (Goulson et al. 2015), the aim of this research was to investigate how the diet of honeybees together with exposure to the neonicotinoid thiacloprid influence their behaviour. With food choice assays, we studied if different food sources lead to a change in bee behaviour when exposed to sublethal doses of thiacloprid. The underlying hypothesis is that pesticide exposure would reduce bee mobility and lead to an increased interest in complex food, as the animals are weakened by the toxic compounds. Individuals fed with highly diverse food resources are expected to be more resistant against pesticide exposure. Generally, we expected the bees to prefer polyfloral honey and pollen over other options as they are assumed to have more balanced nutritional quality than monofloral honey or pollen (Branchiccela et al. 2019). Beside food preference, we also monitored the combined effects of diet and pesticide exposure on bee mobility and feeding activity.

\section{MATERIAL AND METHODS}

\subsection{Experimental set-up, animals, and feeding regimes}

We purchased queens with starter hives of the subspecies Apis mellifera carnica from the Steirische Imkerschule in June 2019. Each colony was placed in a small hive box $(30 \times 30 \times 17 \mathrm{~cm})$ made from polystyrene with six wooden frames in it. All hives were habituated in the bee yard of the University of Graz campus. The rearing resulted in twelve hives, which we split into three groups with different feeding regimes starting on July 9: we fed four hives conventional sugar solution Apiinvert® (a liquid bee food by the German company Südzucker consisting solely of sucrose and its components glucose and fructose), while four hives received monofloral honey (creamy sunflower honey) and the remaining four hives were fed polyfloral honey (a 50-50 mixture of forest and blossom honey), both purchased from the Steirische Imkerschule in Graz. The honeys were diluted so that all three food sources had $70 \%$ sugar concentration. The bees were fed in their outdoor hives with the different food sources for 10 days ad libitum, after which no more food was added to the hives. The bees used in the experiments were extracted from the hives 2 days later, which should be enough time to ensure they had been exposed to the hive specific diet.

\subsection{Thiacloprid exposure}

For the thiacloprid exposure, 30 bees per hive are collected directly from the brood comb with tweezers and transferred into treatment boxes (15 $\times 12 \times 8.5 \mathrm{~cm}$ with a volume of 1.51 ), where they were fed water and thiacloprid in their pretreatment food (sugar solution, monofloral honey, polyfloral honey) via two different syringes, each with a volume of $20 \mathrm{ml}$, for $24 \mathrm{~h}$ ad libitum (Suppl. Fig. 1). As for the control, the same procedure was done without thiacloprid for each of the studied hives. During the 24-h insecticide exposure, the bees were kept in an incubator at $34{ }^{\circ} \mathrm{C}$ and relative humidity above $50 \%$ to simulate normal hive conditions (Williams et al. 2013). We used a sublethal thiacloprid concentration of $6.5 \mu \mathrm{g} / \mathrm{ml}$ food $(6.5 \mathrm{ppm})$. This concentration is about a thousand times higher compared to mean residue levels found in nectar after applying the pesticide as foliar spray on oilseed rape crops in Poland (Pohorecka et al. 2012). However, natural exposure can momentarily be as high as this. For example, the thiacloprid formulation Calypso® has a suggested spraying concentration of 180 $\mu \mathrm{g} / \mathrm{ml}$. Our 24-h exposure with a concentration of $6.5 \mu \mathrm{g} / \mathrm{ml}$ thus reflects a conceivable short time 
exposure to thiacloprid after spraying. Previously, slightly lower concentrations have been used in exposure experiments, but with longer exposure times, for example, a concentration of $5.1 \mu \mathrm{g} / \mathrm{ml}$ for 10 days (Vidau et al. 2011), $3.78 \mu \mathrm{g} / \mathrm{ml}$ for 11 days (Dickel et al. 2018), or $2 \mu \mathrm{g} / \mathrm{ml}$ for 7 and 13 days (Brandt et al. 2016; Liu et al. 2020). Thiacloprid was obtained as dry powder (Sigma) and was first dissolved in dimethyl sulfoxide (DMSO) to create a stock solution. The thiacloprid stock was then used with the same sugar and honey solutions that were used for hive feeding to create a feeding solution with $6.5 \mathrm{ug} / \mathrm{ml}$ thiacloprid. DMSO concentration was adjusted to $0.1 \%$ in all treatments.

\subsection{Behavioural observations}

After the thiacloprid exposure, we carefully opened the boxes and used the first ten individuals per box that were capable of flying out from the boxes towards a window in the lab, where they could be easily caught. This technique ensured that we did not use the individuals which were totally paralyzed by the pesticide, and was deemed to be the least stressful method of capturing, both for the bees and the researchers. We transferred the ten bees individually into $15-\mathrm{ml}$ falcon tubes and starved them for $2 \mathrm{~h}$ at the 34 ${ }^{\circ} \mathrm{C}$ in the incubator. The bees were then transferred one by one into petri dishes for the 10min filming period of the food choice assays.
One filming event was composed of six individual bees in six separate petri dishes from three different hives (one per hive from control and treatment groups) with randomly attached labels to ensure a blinded process. We used Sony Camcorder (model HDR-XR155E) to shoot the videos. Between each take, the whole set-up was rotated 90 degrees to ensure that the direction of the light from the windows did not affect the results.

\subsubsection{Honey choice assay}

For the honey choice assay, we placed each bee in the middle of a petri dish, which was visually divided into three different sectors (A-C). In each sector, we offered a different type of food in the caps of 1.5-ml Eppendorf Tubes ${ }^{\circledR}$ (see Fig. 1). The individuals could choose between their pretreatment food sources: Apiinvert ${ }^{\circledR}$ sugar solution in sector A, monofloral sunflower honey in sector $\mathrm{B}$, and polyfloral honey in sector $\mathrm{C}$.

\subsubsection{Pollen choice assay}

The set-up for the pollen choice assay was identical to the honey choice assay except we used different individuals and offered them sugar-yeast dough (in sector A), monofloral pollen from Sinapis sp. (in sector B), and polyfloral pollen from different local plant sources (in sector $\mathrm{C}$ ). Both the pollen and the honey were purchased at the Steirische Imkerschule. Yeast was used

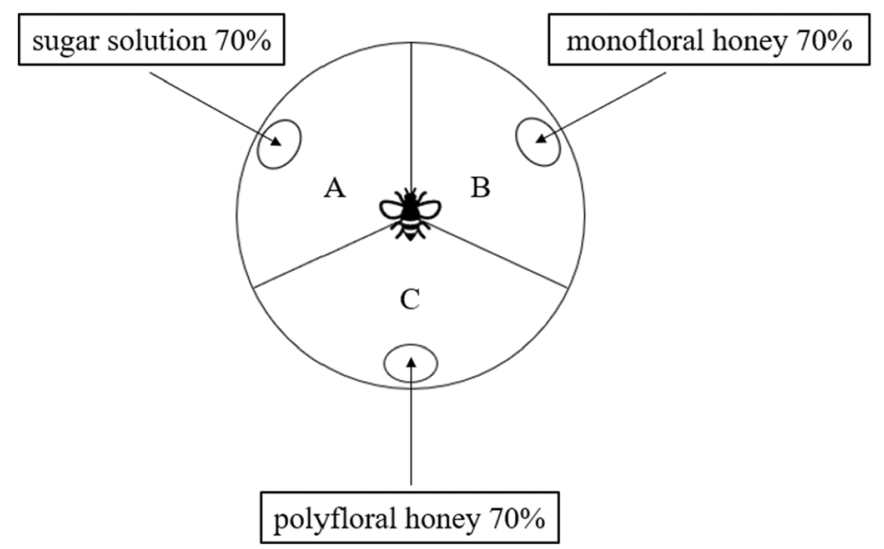

Fig. 1 Experimental set-up for the honey choice assay: A petri dish visually divided into three equally sized sectors with a different food resource in each sector (sector A: sugar solution; sector B: monofloral honey; sector C: polyfloral honey) 
because it has a similar protein concentration but less of the other nutrients present in pollen and is standardly used as a pollen substitute (Westendorp 2015). The different food sources are mixed with sugar and water to make their drinking consistencies equal (Supplementary Table 1 1).

\subsection{Video analyses}

To quantify bee behaviour, we scored $10 \mathrm{~min}$ of the videos with fully blinded procedures regarding the diet and pesticide treatments, starting from when the bee was placed into a petri dish, using the software Jwatcher version 1.0 (Blumstein and Daniel 2007). We recorded the position of the bees in the petri dishes (sector A, $\mathrm{B}$, or $\mathrm{C}$ ), and whether the individuals were consuming the offered food or not (proboscis in food) by pressing predefined keystroke combinations (e.g. pressing A when the bee was in sector A, and $\mathrm{B}$ when it changed to sector B). The software simultaneously recorded the corresponding times to each behaviour. This way we could identify the favoured food source of the examined individual (where it ate longest) and its mobility, defined by the number of times the individual crossed the lines between sectors.

\subsection{Statistical analyses}

For data analysis, we used $\mathrm{R}$ version 3.6.1 ( $\mathrm{R}$ Development Core Team). The analyses for the honey and the pollen assays were done separately. To assess the effects of the whole pre-experimental diet variable instead of the effects of every factor level separately, we produced an ANOVA results table for each analysis (using the 'Anova.clmm' function from the package "RVAideMemoire" in case of Cumulative Link Mixed-effects Model (CLMM), and 'Anova' function from the package "car" in the rest).

A) Food preference To determine factors influencing which food bees favoured, we ran a fullfactorial CLMM with "clmm" function from the “ordinal” package (Christensen 2015, 2019). We excluded the bees which did not eat at all during the experiments $[n$ (honey assay) $=18, n$ (pollen assay $)=38]$. We included the favoured food (which food bees spent most time eating) as the ordinal response variable (in the order of sugar/ yeast $<$ monofloral $<$ polyfloral), and used treatment (pesticide/control) and pre-experimental diet (sugar solution/monofloral honey/polyfloral honey) as explanatory variables, and researchers scoring the videos and nest IDs as random effects. As the interaction between treatment and preexperimental diet was not statistically significant in either of the models, we excluded it from the final models.

B) Feeding activityBecause there were 56 bees in total that did not eat at all, we investigated if the treatment and pre-experimental diet affected whether bees ate or not. We formed a full-factorial generalized mixed-effects model (GLMM) with binomial distribution, using the "glmer" function from the package "lme4" (Bates et al. 2014). We included the eating status (did eat/did not eat) as the response variable, treatment (pesticide/control) and preexperimental diet (sugar solution/monofloral honey/polyfloral honey) as the explanatory variables, and researchers scoring the videos and nest IDs as random effects. As the interactions between treatment and preexperimental diet were not statistically significant, we excluded them from the final models.

C) Mobility To analyse whether pesticide and preexperimental diet affected bee mobility (the total number of times crossing a line between sectors), we ran a full-factorial generalized mixed-effects model (GLMM) with "glmer" function from the "lme4" package (Bates et al. 2014). The analyses were run separately for pollen choice and honey choice assays, as their different environmental context likely affects bee behaviour, too, but is not the subject of our study. We fit the mobility as the response variable, treatment (pesticide/control) and pre-experimental diet (sugar solution/ monofloral honey/polyfloral honey) as explanatory variables, and researchers scoring the videos and nest IDs as random effects. We ran the analyses first with Poisson distributions, but since they were over-dispersed (the square root of the 
penalized residual sum of squares divided by the number of observations: 5.94 and 7.88, respectively), we did the final analyses with negative binomial distribution.

\section{RESULTS}

From 366 control bees, one died during the 24$\mathrm{h}$ pesticide exposure $(0.3 \%)$. The treatment group consisted of 365 individuals, of which three bees $(0.8 \%)$ died. This low number of dead bees shows that pesticide treatment had no effect on honeybee survival.

A)Food preferencePesticide treatment significantly affected the choice between different honeys (CLMM: $\left.\chi^{2}=4.436, n=198, \mathrm{df}=1, p=0.035\right)$. Monofloral honey is clearly favoured by control bees that had not been treated with pesticides, whereas pesticide-treated bees change their choice towards polyfloral honey (Fig. 2a). Preexperimental diet did not affect the honey choice (CLMM: $\chi^{2}=3.286, n=198, \mathrm{df}=2, p=0.193$ ). In the pollen assay, neither treatment (CLMM: $\chi^{2}$ $=0.002, n=201$, df $=1, p=0.96$ ) nor preexperimental diet (CLMM: $\chi^{2}=0.684, n=201$, df $=2, p=0.71$ ) affected the food choice. Polyfloral pollen is overall the most favoured food for both control and pesticide-treated bees $(68 \%$; Fig. 2b).

B)Feeding activity Neither pesticide nor preexperimental diet affected whether bees ate or not in either of the food assays (honey GLMM: treatment, $\chi 2=0.97, \mathrm{df}=1, p=0.33$ and preexperimental diet, $\chi 2=2.46, \mathrm{df}=2, p=0.29 ; n=$ 216; pollen GLMM: treatment, $\chi 2=0.0024$, df $=$ $1, p=0.96$ and pre-experimental diet, $\chi 2=1.86$, $\mathrm{df}=2, p=0.40 ; n=239)$. The number of bees not eating at all ranged between 5.6 and $20 \%$ in the treatment and pre-experimental diet groups (Supplementary Table 2).

Bees in the honey choice assay eat primarily in the beginning of the experiment, while bees in the pollen choice assay eat repeatedly during the whole filming period and obviously prefer polyfloral pollen (control and treatment) (Suppl. Fig. 2).
C)Mobility The pesticide treatment mostly reduced bee mobility, but the effect depended on the previous diet in both the honey assays (GLMM with negative binomial: $\chi^{2}=6.41, \mathrm{df}=2, n=216, p=$ 0.041 ) and pollen assays (GLMM with negative binomial: $\chi^{2}=6.15$, df $=2, n=239, p=0.046$ ). The full results on bee mobility are presented in Supplementary Tables 3 and 4.

In the honey choice assay, mobility of pesticidetreated bees (mean number of times crossing the sector line) was lower than that of control bees, if they had eaten polyfloral honey before the experiment, but it did not differ between the treatments if bees had eaten other food (Fig. 3a, Supl. Table 4). In the pollen assay, mobility of pesticide-treated bees is lower than that of control bees when their previous diet was monofloral honey (Fig. 3b, Supl. Table 4). The pesticidetreated bees with sugar solution diet had slightly higher mobility than control bees.

\section{DISCUSSION}

In our study, pesticide and diet had combined effects on bee mobility. We also found out that pesticide exposure changed bee food preference towards polyfloral honey, while bees always preferred polyfloral pollen. These results are in accordance with other findings in pollinators, which show, for example, that bumblebees best thrive on polyfloral pollen (Dance et al. 2017), and poor diet in combination with pesticides has negative effects on survival in both bees and bumblebees (Dance et al. 2017; Tosi et al. 2017).

Overall, we show that pesticide exposure leads to different changes in bee mobility based on their previous diets. Pesticide exposure led to lower mobility for the bees fed on monofloral honey, but at the same time, bees fed on sugar solution were more mobile in our pollen choice assay. Interestingly, the mobility results in the honey choice assay are yet slightly different. This shows that the coeffect of diet and pesticide exposure, while it clearly exists, is not simple. These results indicate that nutrition is a decisive factor influencing the susceptibility against pesticides and other detrimental environmental impacts, as confirmed also in the study by Archer et al. (2013). The 


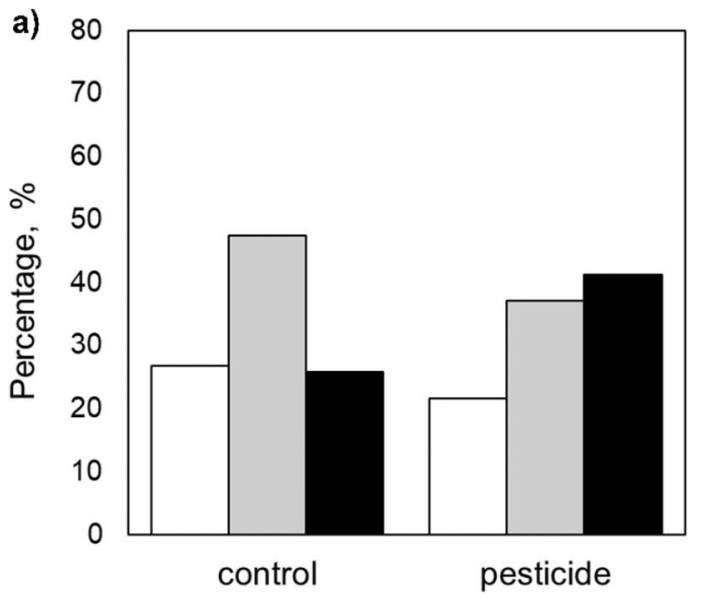

Favored food

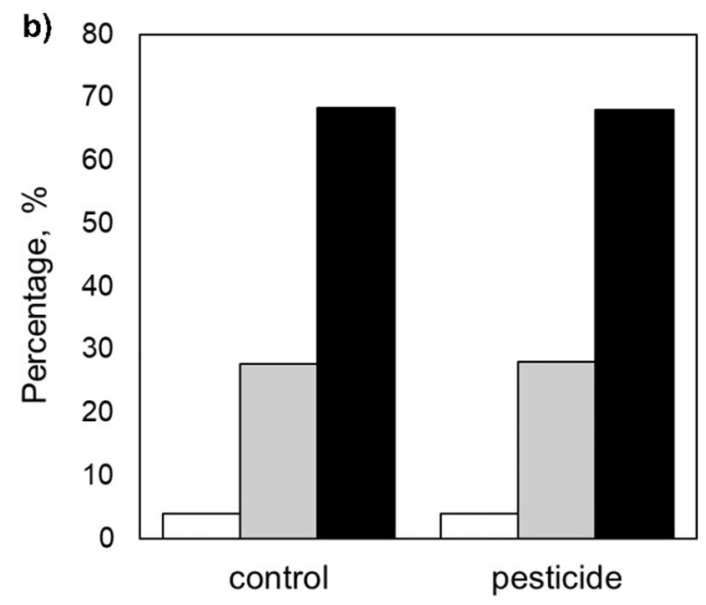

Favored food

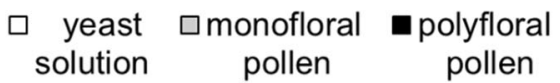

Fig. 2 The percentage of bees favouring (eating the longest time) each food source within both treatment groups in (a) the honey choice assay and (b) the pollen choice assay

harmfulness of neonicotinoids may be influenced by the health status of individual bees and the whole colony. For example, Vidau et al. (2011) shows that the mortality of honeybees infected with Nosema ceranae (a parasite) was significantly higher when combined with thiacloprid

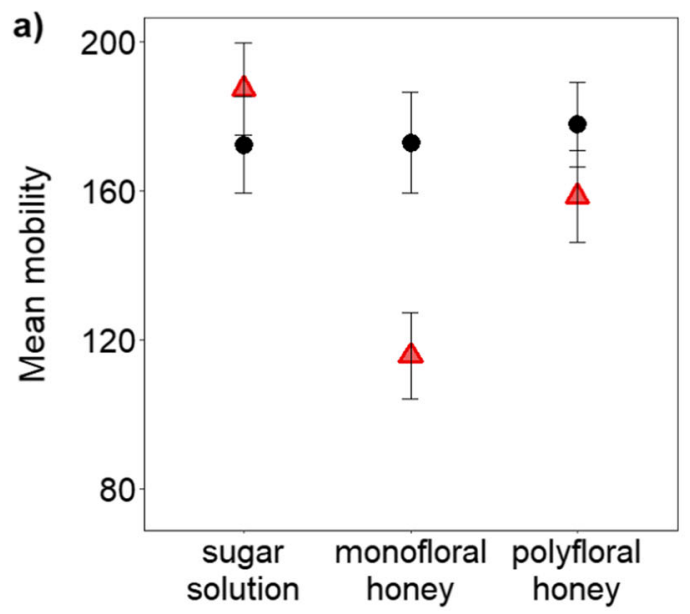

Diet exposure $(71 \%)$ compared to the parasite infection alone $(47 \%)$. Our results, together with previous findings, support the idea that the combined effects of different stressors can have more severe consequences on bees than each of these stressors alone.

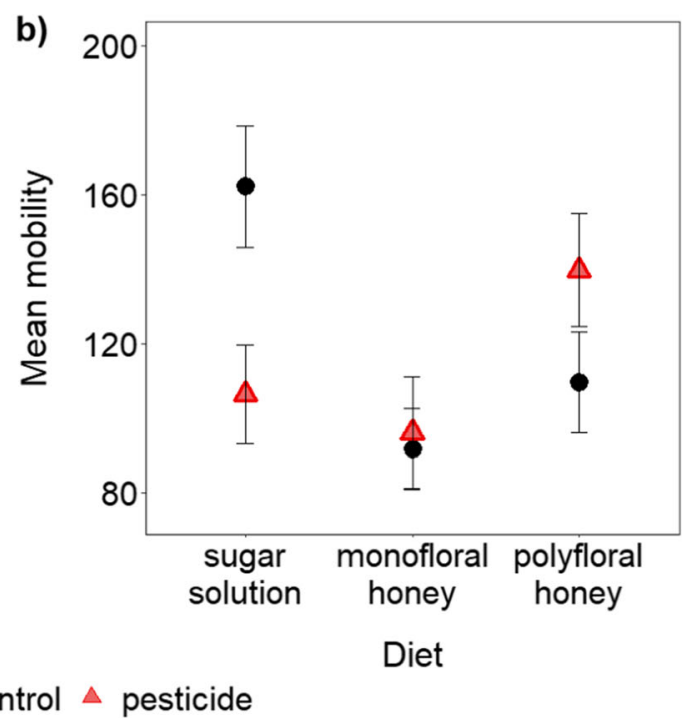

Fig. 3 Effects of pesticide treatment and pre-experimental diet on mean bee mobility (mean of the total number of times crossing a line between sectors \pm SE) in (a ) honey choice assay and (b ) pollen choice assay. Bees had either sugar solution, monofloral honey, or polyfloral honey diets before the expeiment 
A study of Hendriksma and Shafir (2016) revealed that bees can discriminate between different diets and choose complementary food sources to prevent nutritional deficits. Several insects have shown to have the ability to selfmedicate (Abbot et al 2009; Bos et al 2015) and reduce stressor-related effects on the health. We did not analyse the nutritional value of the different food sources in the present study, but based on earlier studies, we can assume that polyfloral honey and pollen constitute a more balanced and varied diet, while sugar and yeast or monofloral pollen and honey represent more one-sided nutrition (Brodschneider and Crailsheim 2010; Vaudo et al. 2015). As nutrition probably plays a role in insecticide resistance (Kramer et al. 1990), the bees may need to look for more nutrientrich diet to meet their needs. We found that thiacloprid treatment changes bees' food choices towards polyfloral honey, presumably because it is more nutrient rich and enables a stronger baseline immunocompetence and general fitness (Alaux et al. 2010). There is no similar effect on pollen choice because bees preferred polyfloral pollen independent of treatment. The consumption of high diversity pollen improves the tolerance against pesticides (Schmehl et al. 2014), and an inadequate supply with pollen reduces stress resistance and may cause colony collapse disorder (Zachary 2012).

Even though previous diet can affect insects' further food choices, for example, through them compensating for lack of nutrients in the earlier diet (Berner et al. 2005, Hendriksma and Shafir 2016), or by them favouring familiar food (Costa et al. 2016), the previous diet in our study had no effect on the food choices during the experiments. The bees did not, for example, prefer the food they were given before the experiments. Instead, they had an overall preference for honey over sugar and a strong preference for polyfloral pollen. They seem to prefer more complex food, regardless of what they had before, likely due to evolutionary adaptations. If we had exposed the hives to different diets for a longer time, we might have seen some effects of diet on their further food choices, as has been shown on other insects (Berner et al. 2005, Costa et al. 2016). In our experiment, the bees were also allowed to forage freely during the diet treatment, making their diets more similar, although they always consumed the food in the hives first.

It is well-known that neonicotinoids have detrimental effects for honeybees and other pollinators, and even low concentrations of thiacloprid severely impair the cognitive abilities of honeybees, like navigation and memory, as well as the motivation to forage and to communicate their findings (Wood and Goulson 2017; Muth et al. 2019; Siviter et al. 2018). The study of Tison et al. (2016) showed that foraging bees chronically exposed to sublethal doses of thiacloprid (4.5 ppm) consumed less food, performed less waggle dances, and showed increased problems of finding back to their hives compared to control groups. Our survey confirms these results, even in the study set-up where the effects of impaired flight are ruled out. Bee behaviour and food choice are affected by pesticides even when the food is directly in front of them and easily accessible. The effect is strong enough to be shown even with our limited colony-level sample size. Given the large amount of colonylevel variation in social bees, further studies on this topic, and especially its importance in natural populations, are gravely needed.

We can conclude that thiacloprid significantly affected the food choice in the honey choice assay, while pre-experimental diet had no influence in our study. As bees treated with thiacloprid preferred the food with higher nutritional value (polyfloral), our findings suggest that bees make food choices dependent on their current health status. Concerning mobility, the effect of pesticide depended on the previous diet, which is a novel result and needs further inspection. Overall, our results indicate that the diet is an important factor influencing the susceptibility to pesticides. Monitoring the interactions between different stressors on pollinators is of tremendous importance because it will help to better understand whether they act additively or 
antagonistically (Collison et al. 2016) and how they influence pollinator health and thus the functioning of the whole ecosystem.

\section{SUPPLEMENTARY INFORMATION}

The online version contains supplementary material available at https://doi.org/10.1007 /s13592-021-00853-x.

\section{ACKNOWLEDGEMENTS}

We thank our institute's beekeeper Mag. Jutta Vollmann, who offered invaluable assistance with maintaining the bee colonies and handling the bees. We also thank Unni Pulliainen who participated to the planning of this work, and the University of Graz for providing their facilities.

\section{AUTHORS' CONTRIBUTIONS}

$\mathrm{SH}$ and DF conceived this study. $\mathrm{CV}, \mathrm{SH}, \mathrm{ML}$, and $\mathrm{AMB}$ designed and performed the experiments, analysed the results, and wrote the manuscript. DF participated in the redaction and revision of the article.

\section{FUNDING}

Open access funding provided by University of Graz. The work was funded by Finnish Cultural Foundation (00180246 to Dalial Freitak) and the Entomological Association in Helsinki (to Sanja Maria Hakala).

\section{DATA AVAILABILITY}

The data associated the manuscript is deposited to the online data depository, and can be found under following link:

\section{DECLARATIONS}

Ethics approval Not applicable

Code availability Not applicable

Conflict of interest Authors declare no competing

\section{OPEN ACCESS}

This article is licensed under a Creative Commons Attribution 4.0 International License, which permits use, sharing, adaptation, distribution and reproduction in any medium or format, as long as you give appropriate credit to the original author(s) and the source, provide a link to the Creative Commons licence, and indicate if changes were made. The images or other third party material in this article are included in the article's Creative Commons licence, unless indicated otherwise in a credit line to the material. If material is not included in the article's Creative Commons licence and your intended use is not permitted by statutory regulation or exceeds the permitted use, you will need to obtain permission directly from the copyright holder. To view a copy of this licence, visit http://creativecommons. org/licenses/by/4.0/.

\section{Author Information}

https://datadryad.org/stash/share/5wWStN8WZY6q9 dzCGc5tIHm6ChKerwsalZUROypMwKI.

Effets conjoints du régime alimentaire et de l'exposition aux néonicotinoïdes sur la mobilité des abeilles et le choix des aliments.

thiaclopride / Apoidea / préférence alimentaire / malnutrition / comportement.

Gemeinsame Effekte von Futter und NeonikotinoidExposition auf die Mobilität und Futterwahl von Honigbienen.

Thiacloprid / Apoidea / Futterpräferenz / Mangelernährung / Verhalten.

\section{REFERENCES}

Alaux, C., Ducloz, F., Crauser, D. \& Le Conte, Y., 2010. Diet Effects on Honeybee Immunocompetence, London, UK: Biol Lett, 6(4):562-565, https://doi. org/10.1098/rsbl.2009.0986.

Archer, C. R., Pirk, C. W., Wright, G. A. \& Nicolson, S. W., 2013. Nutrition affects survival in African honeybees exposed to interacting stressors, s.1.: Funct Ecol; 28 (4):913-923; https://doi.org/10.1111/13652435.12226 . 
Bates, D. M., Mächler, M., Bolker, B., Walker, S., 2014. Fitting Linear Mixed-Effects ModelsUsinglme4 . Journal of Statistical Software, $67(1): 1-48$. https://doi. org/10.18637/jss.v067.i01.

Berner, D., Blanckenhorn, W. U., Körner, C., 2005. Grasshoppers cope with low host plant quality by compensatory feeding and food selection: $N$ limitation challenged. OIKOS $111: 525-533$.

Blumstein, D. T. \& Daniel, J. C., 2007. Quantifying Behavior the Jwatcher Way. Sunderland: Sinauer Associates.

Branchiccela, B. et al., 2019. Impact of nutritional stress on the honeybee colony health, Montevideo, Uruguay: Sci Rep, 9(1):10156, https://doi.org/10.1038/s41598019-46453-9.

Brandt, A., Gorenflo, A., Siede, R., Meixner, M., and Büchler, R., 2016. The neonicotinoids thiacloprid, imidacloprid, and clothianidin affect the immunocompetence of honey bees (Apis mellifera L.). J Insect Physiol, 86:40-47. https://doi.org/10.1016/j. jinsphys.2016.01.001.

Brodschneider, R. \& Crailsheim, K., 2010. Nutrition and health in honey bees, Karl-Franzens-University Graz, Austria: Apidologie; 41 (3):278-294, https://doi. org/10.1051/apido/2010012.

Collison, E., Hird, H., Cresswell, J. \& Tyler, C., 2016. Interactive effects of pesticide exposure and pathogen infection on bee health - a critical analysis, Cambridge, UK: Biol Rev, 91 (4):1006-1019. https://doi. org/10.1111/brv.12206.

Craddock, H. A. et al., 2019. Trends in neonicotinoid pesticide residues in food and water in the United States, 1999-2015, s.1.: Environ Health; 18(1):7; https://doi.org/10.1186/s12940-018-0441-7.

Christensen, H. R. B., 2015. Analysis of ordinal data with cumulative link models-estimation with the $R$ package ordinal. Retrieved from https://mran. microsoft.com/snapshot/2015-03-12/web/packages/ ordinal/vignettes/clm_intro.pdf.

Christensen, R. H. B., 2019. Package 'ordinal' . Retrieved from https://cran.rproject.org/web/packages/ ordinal/ordinal.pdf.

Costa, T. M., Hebets, E. A., Melo, D., Willemart R.H., 2016. Costly learning: preference for familiar food persists despite negative impact on survival. Biology Letters 12 (7):20160256, https://doi.org/10.1098 /rsbl.2016.0256.

Dance, C., Botías, C. \& Goulson, D., 2017. The combined effects of a monotonous diet and exposure to thiamethoxam on the performance of bumblebee micro-colonies, s.1.: Ecotoxicol Environ Saf; 139:194201; https://doi.org/10.1016/j.ecoenv.2017.01.041.

Di Pasquale, G. et al., 2013. Influence of Pollen Nutrition on Honey Bee Health: Do Pollen Quality and Diversity Matter?, Avignon, France: PLoS One, 8(8): e72016, https://doi.org/10.1371/journal.pone.0072016.

Dickel, F., Münch, D., Amdam, G. V., Mappes, J., and Freitak, D., 2018. Increased survival of honeybees in the laboratory after simultaneous exposure to low doses of pesticides and bacteria. PLoS One, 13. https://doi.org/10.1371/journal.pone.0191256.

Dolezal, A. G. \& Toth, A. L., 2018. Feedbacks between nutrition and disease in honey bee health, University of Illinois, United States: Curr Opinion Insect Sci, 26:114-119, https://doi.org/10.1016/j. cois.2018.02.006.

Goulson, D., 2013. REVIEW: An overview of the environmental risks posed by neonicotinoid insecticides, University of Stirling, Stirling, UK: J Appl Ecol, 50 (4):977-987, https://doi.org/10.1111/13652664.12111.

Goulson, D., Nicholls, E., Botías, C. \& Rotheray, E. L., 2015. Bee declines driven by combined stress from parasites, pesticides, and lack of flowers, Washington, D.C.: Science, 347 (6229):1255957, https://doi. org/10.1126/science. 1255957 .

Hendriksma, H. P. \& Shafir, S., 2016. Honey bee foragers balance colony nutritional deficiencies, Bee Research Center, The Hebrew University of Jerusalem, Israel: Behav Ecol Sociobiol, 70 :509-517.

Henry, M. et al., 2012. A Common Pesticide Decreases Foraging Success and Survival in Honey Bees, Washington, D.C.: Science, 336 (6079):348-350, https://doi. org/10.1126/science. 1215039 .

Kramer, R. D., Koehler, P. G., Patterson, R. S., Slansky, F., 1990. Nutritional Status and Insecticide Tolerance in German Cockroaches (Orthoptera: Blattellidae). Journal of Economic Entomology, 83 (5):1912-1917. https://doi.org/10.1093/jee/83.5.1912.

Kremen, C. et al., 2007. Pollination and Other Ecosystem Services Produced by Mobile Organisms: A Conceptual Framework for the Effects of Land-Use Change, University of California, Berkeley: Ecol Lett, 10 (4):299-314, https://doi.org/10.1111/j.14610248.2007.01018.x..

Liu, Y. J., Qiao, N. H., Diao, Q. Y., Jing, Z., Vukanti, R., Dai, P. L., et al. 2020. Thiacloprid exposure perturbs the gut microbiota and reduces the survival status in honeybees. J Hazard Mater, 389 :121818. https://doi. org/10.1016/j.jhazmat.2019.121818.

Muth, F., Francis, J. S. \& Leonard, A. S., 2019. Modalityspecific impairment of learning by a neonicotinoid pesticide, London, UK: Royal Soc, 15(7), https://doi. org/10.1098/rsbl.2019.0359.

Pohorecka, K. et al., 2012. Residues of Neonicotinoid Insecticides in Bee Collected Plant Materials from Oilseed Rape Crops and their Effect on Bee Colonies, National Veterinary Research Institute, Puławy, Poland: J Apicult Sci, 56(2), https://doi.org/10.2478 /v10289-012-0029-3.

Requier, F. et al., 2018. Trends in beekeeping and honey bee colony losses in Latin America, s.1.: J Apic Res, 57 (5): 657-662, https://doi.org/10.1080 /00218839.2018.1494919.

Schmehl, D. R., Teal, P. E., Frazier, J. L. \& Grotzinger, C. M., 2014. Genomic analysis of the interaction between pesticide exposure and nutrition in honey bees (Apis mellifera), s.1.: J Insect Physiol; 71:177-190; https://doi.org/10.1016/j.jinsphys.2014.10.002. 
Siviter, H., Koricheva, J., Brown, M. J. F. \& Leadbeater, E., 2018. Quantifying the impact of pesticides on learning and memory in bees, London, UK: J Appl Ecol, 55 (6): 2812-2821, https://doi.org/10.1111/1365-2664.13193.

Steinhauer, N. et al., 2018. Drivers of colony losses, s.1.: Curr Opinion Insect Sci, $26: 142-148$. https://doi. org/10.1016/j.cois.2018.02.004.

Tison, L. et al., 2016. Honey Bees' Behaviour Is Impaired by Chronic Exposure to the Neonicotinoid Thiacloprid in the Field, Washington, D.C.: Environ Sci Technol, 50 (13):7218-7227, https://doi.org/10.1021/acs.est.6 b02658.

Tosi, S., Nieh, J. C. \& Sgolastra, F., 2017. Neonicotinoid pesticides and nutritional stress synergistically reduce survival in honey bees, s.1.: Proc R Soc B Biol Sci; 284 (1869):20171711; https://doi.org/10.1098 /rspb.2017.1711.

van Engelsdorp, D. \& Meixner, M. D., 2010. A historical review of managed honey bee populations in Europe and the United States and the factors that may affect them, s.1.: J Invertebr Pathol, 103:S80-S95, https://doi. org/10.1016/j.jip.2009.06.011.

Vaudo, A. D., Tooker, J. F., Grozinger, C. M. \& Patch, H. M., 2015. Bee nutrition and floral resource restoration, s.1.: Curr Opinion Insect Sci; 10:133-141; https://doi.org/10.1016/j.cois.2015.05.008.

Vidau, C. et al., 2011. Exposure to Sublethal Doses of Fipronil and Thiacloprid Highly Increases Mortality of Honeybees Previously Infected by Nosema ceranae,
Clermont Université Aubière, France: PLoS One, 6 (6): e21550, https://doi.org/10.1371/journal. pone. 0021550 .

Westendorp, P. v., 2015. Pollen Substitutes and Supplements, British Columbia: Ministry of Agriculture; Plant \& Animal Health Branch.

Williams, G. R., Alaux, C., Costa, C., Csáki, T., Doublet, V., Eisenhardt, D., et al. 2013. Standard methods for maintaining adult Apis mellifera in cages under in vitro laboratory conditions. J Apic Res, 52:1-36, https://doi.org/10.3896/IBRA.1.52.1.04.

Wood, T. J. \& Goulson, D., 2017. The environmental risks of neonicotinoid pesticides: a review of the evidence post 2013, s.1.: Environ Sci Pollut Res; 24 (21):1728517325; https://doi.org/10.1007/s11356-017-9240-x.

Yang, E., Chuang, Y. \& Chang, L., 2008. Abnormal Foraging Behavior Induced by Sublethal Dosage of Imidacloprid in the Honey Bee (Hymenoptera: Apidae), Oxford, UK: J Econ Entomol, 101 (6):17431748, https://doi.org/10.1603/0022-0493-101.6.1743.

Zachary, H., 2012. Pollen nutrition affects honey bee stress resistance, s.1.: Terrestrial Arthropod Rev; 5:175189; https://doi.org/10.1163/187498312X639568.

Publisher's note Springer Nature remains neutral with regard to jurisdictional claims in published maps and institutional affiliations. 